$2016 / 10$

Stable Sets in Matching Problems with Coalitional Sovereignty and Path Dominance

P. JeAn-Jacques HeRings, Ana MaUleon and Vincent VANNETELBOSCH

\title{
50 YEARS OF
}

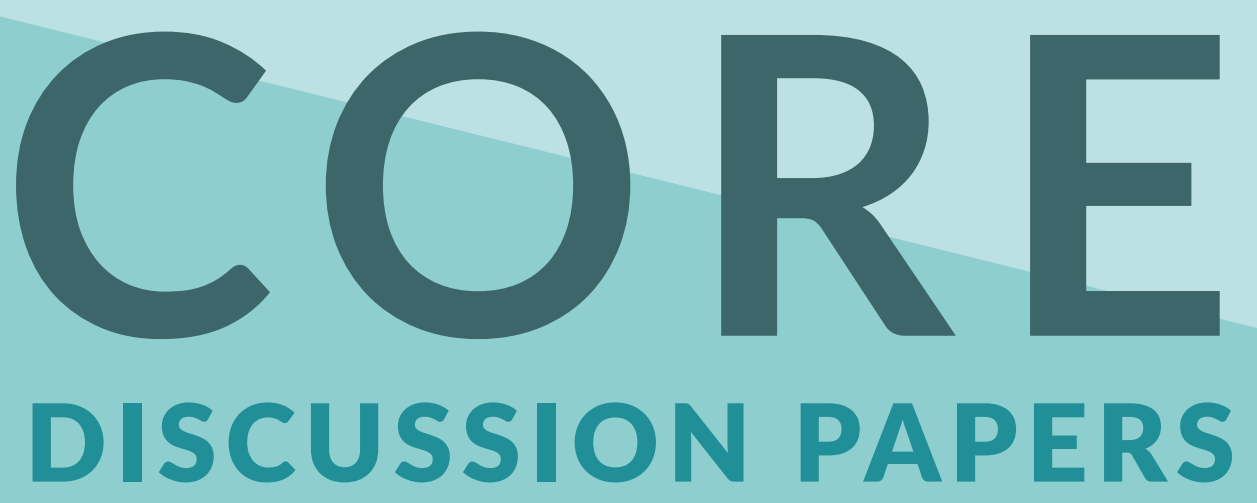




\section{CORE}

Voie du Roman Pays 34, L1.03.01

Tel (32 10) 474304

Fax (32 10) 474301

Email: immaq-library@uclouvain.be

http://www.uclouvain.be/en-44508.html 


\title{
Stable Sets in Matching Problems with Coalitional Sovereignty and Path Dominance
}

\author{
P. Jean-Jacques Herings*
}

\author{
Ana Mauleon ${ }^{\dagger}$
}

Vincent Vannetelbosch ${ }^{\ddagger}$

April 20, 2016

\begin{abstract}
We study von Neumann Morgenstern stable sets for one-to-one matching problems under the assumption of coalitional sovereignty, meaning that a deviating coalition of players does not have the power to arrange the matches of agents outside the coalition. We study both the case of pairwise and coalitional deviations. We argue further that dominance has to be replaced by path dominance along the lines of van Deemen (1991) and Page and Wooders (2009). This results in the pairwise myopic vNM set and the myopic vNM set, respectively. We obtain a unique prediction for both types of stable sets: the set of matchings that belong to the core. We also show that the pairwise and coalitional analogues of the level- 1 farsighted set yield the core as the unique prediction.
\end{abstract}

Key words: Matching problems, stable sets, coalitional sovereignty.

JEL classification: C70, C78.

*Department of Economics, Maastricht University, Maastricht, The Netherlands. E-mail: P.Herings@maastrichtuniversity.nl

${ }^{\dagger}$ CEREC, Saint-Louis University - Brussels; CORE, University of Louvain, Louvain-la-Neuve, Belgium. E-mail: ana.mauleon@usaintlouis.be

${ }^{\ddagger}$ CORE, University of Louvain, Louvain-la-Neuve, Belgium. E-mail: cent.vannetelbosch@uclouvain.be 


\section{Introduction}

In the one-to-one matching model known as the marriage problem, there are two disjoint sets of agents, say men and women. The problem is to match agents from one side of the market with agents from the other side, whereas each agent also has the possibility of remaining single. We refer to Roth and Sotomayor (1990) for a comprehensive overview on two-sided matching problems.

For marriage problems, stability is considered to be a central property. A matching is stable if each agent on one side is matched with an acceptable agent on the other side and no two agents of different sides would prefer to be matched to each other rather than to

stick to their current situation. For marriage markets, this stability notion is known to be equivalent to core stability.

A matching is in the core if there is no subset of agents who, by forming only partnerships among themselves, can all obtain a strictly preferred outcome. Gale and Shapley (1962) have shown that the core of a marriage problem is non-empty. Although elements of the core have the property that they are stable once reached, it depends on the underlying environment whether it is possible to reach some core element from any initial situation. Stable sets as defined in von Neumann and Morgenstern (1944) address this concern.

A stable set is a set of outcomes that satisfies internal and external stability. As argued by von Neumann and Morgenstern (1944), p. 41, a stable set describes the "established order of society" or "accepted standard of behavior." Internal stability "expresses the fact that the standard of behavior is free from inner contradictions". External stability "can be used to discredit any non-conforming procedure".

vNM stable sets are crucially dependent on the concept of dominance. Under the standard definition, a matching is dominated by another matching if there is a coalition such that all its members prefer the latter matching to the former and no coalition member has a partner outside the coalition. A set of matchings is a vNM stable set if it satisfies the conditions of internal and external stability with respect to this dominance relation. Internal stability requires that no matching inside the set is dominated by a matching belonging to the set. External stability imposes that any matching outside the set is dominated by some matching belonging to the set. Ehlers (2007) has shown that for oneto-one matching problems, the set of matchings in the core is a subset of any vNM stable set and a vNM stable set can contain matchings outside the core. The vNM stable set may not be unique.

The standard dominance relation used to define vNM stable sets violates the assumption of coalitional sovereignty, the property that an objecting coalition cannot enforce matches between members outside the coalition. Recently, Ray and Vohra (2015) have criticized the standard definition of the vNM stable set for non-transferable utility games because it 
denies coalitional sovereignty of players outside the objecting coalition. Exactly the same criticism applies to one-to-one matching problems.

A further criticism of the standard definition of the vNM stable set is that it does not take into account that a deviation by a coalition can be followed by further deviations. This corresponds to the well-known critique by Harsanyi (1974) to the vNM stable set. We will follow the approach by van Deemen (1991) and Page and Wooders (2009), which takes into account that if a matching is blocked by some coalition and the resulting matching is not in the stable set itself, then further deviations will take place. This observation leads Van Deemen (1991) to define the generalized stable set for abstract systems and Page and Wooders (2009) to define the stable set with respect to path dominance. We show by means of a simple example that not allowing for path dominance in one-to-one matching problems leads to highly undesirable conclusions. External and internal stability as defined by path dominance also corresponds to the myopic blocking dynamics as introduced by Roth and Vande Vate (1990) for marriage markets and by Jackson and Watts (2002) for network formation problems.

For one-to-one matching problems, the dominance relation satisfying coalitional sovereignty is defined in a straightforward way. If a coalition deviates, then it is free to form any match between its members, it cannot affect existing matches between members outside the coalition, and previous matches between coalition and non-coalition members are destroyed. Using the path dominance relation to define internal and external stability, now referred to as myopic internal stability and myopic external stability, leads to the concept of the myopic vNM set. Since in matching theory it is also often assumed that only pairwise deviations are feasible, we also define the concept of the pairwise myopic vNM set in an analogous way.

Since the myopic and the pairwise myopic vNM sets are based on paths of deviations resulting from the direct dominance relation, we use the adjective myopic. An alternative would be a farsighted approach based on the indirect dominance relation as introduced in Harsanyi (1974) and further developed in Chwe (1994). The von Neumann-Morgenstern farsighted stable sets have been characterized in Mauleon, Vannetelbosch, and Vergote (2011) as the singleton core elements.

We show that there is a unique myopic vNM and a unique pairwise myopic vNM set and that both sets coincide with the core. Although, as shown by Ehlers (2007), the core may not be a vNM stable set under the standard definition of the direct dominance relation, ${ }^{1}$ it turns out to be the unique prediction when coalitional sovereignty and path dominance is taken into account.

\footnotetext{
${ }^{1}$ There are not so many classes of games where the core is the unique vNM stable set of the game. One example of such a class is the class of convex games, see Shapley (1971).
} 
An alternative to myopic vNM sets consists of an adaptation of the level-1 farsighted set of Herings, Mauleon and Vannelbosch (2014) to one-to-one matchings problems. Herings, Mauleon, and Vannetelbosch (2014) define this notion for network formation problems and also allow for higher levels of farsightedness. The definition of the level-1 farsighted set requires level-1 external stability as well as level-1 deterrence of external deviations, and considers a minimal set of matchings satisfying these two properties. Level-1 external stability is equivalent to pairwise myopic external stability. Level-1 deterrence of external deviations requires that there is no pairwise myopic improvement from a matching inside the level-1 farsighted set to a matching outside the set. The minimality requirement can also be viewed as a requirement of internal stability, see also Myerson and Weibull (2015) on this point of view.

Contrary to vNM stable sets, level-1 farsighted sets can be shown to exist under quite general assumptions. For one-to-one matching problems, they again yield the core as the unique prediction. The same prediction follows if the level-1 farsighted set is based on general coalitional deviations rather than pairwise deviations.

The paper is organized as follows. Section 2 introduces one-to-one matching problems and standard notions of stability. Section 3 defines and characterizes both the myopic and the pairwise myopic vNM set. Section 4 studies the level-1 farsighted set. Section 5 concludes.

\section{One-to-One Matching Problems}

A one-to-one matching problem consists of a finite set of individuals $N$, partitioned into a set of men $M$ and a set of women $W$. The set of non-empty subsets of $N$ is denoted by $\mathcal{N}$. Each individual $i \in N$ has a complete and transitive preference ordering $\succ_{i}$ over the agents on the other side of the market and the prospect of being alone. Preferences are assumed to be strict. Let $\succ=\left(\left(\succ_{m}\right)_{m \in M},\left(\succ_{w}\right)_{w \in W}\right)$ be a preference profile. We write $m \succ_{w} m^{\prime}$ if woman $w$ strictly prefers $m$ to $m^{\prime}$. Similarly, we write $w \succ_{m} w^{\prime}$ if man $m$ strictly prefers $w$ to $w^{\prime}$. A one-to-one matching problem is a triple $(M, W, \succ)$.

A matching is a function $\mu: N \rightarrow N$ satisfying the following properties:

(i) For every $m \in M, \mu(m) \in W \cup\{m\}$.

(ii) For every $w \in W, \mu(w) \in M \cup\{w\}$.

(iii) For every $i \in N, \mu(\mu(i))=i$.

The set of all matchings is denoted by $\mathcal{M}$. Given a matching $\mu$, individual $i \in N$ is said to be unmatched or single if $\mu(i)=i$. A matching $\mu$ is individually rational if each 
agent is acceptable to his or her mate, so for every $i \in N$ it holds that $\mu(i) \succ_{i} i$ or $\mu(i)=i$. A matching $\mu$ that is not individually rational can be blocked by an individual with an unacceptable partner. For a given matching $\mu$, a pair $\{m, w\}$ is said to form a blocking pair if $w$ and $m$ are not matched to one another but prefer one another to their mates at $\mu$, i.e. $w \succ_{m} \mu(m)$ and $m \succ_{w} \mu(w)$. A matching $\mu$ is stable if it is not blocked by any individual or any pair of agents.

For every $i \in N$, we extend the preference $\succ_{i}$ over the agent's potential partners to the set of matchings in the following way. We say that agent $i$ prefers the matching $\mu^{\prime}$ to the matching $\mu$ if $\mu^{\prime}(i) \succ_{i} \mu(i)$ and we write $\mu^{\prime} \succ_{i} \mu$. A coalition $S \in \mathcal{N}$ is said to block a matching $\mu \in \mathcal{M}$ if there exists a matching $\mu^{\prime} \in \mathcal{M}$ such that $\mu^{\prime}(S)=S$ and $\mu^{\prime} \succ_{S} \mu$, where $\mu^{\prime} \succ_{S} \mu$ is defined as $\mu^{\prime}(i) \succ_{i} \mu(i)$ for every $i \in S$. Such a matching $\mu^{\prime}$ is said to dominate the matching $\mu$ via $S$. The set of all such matchings is denoted by $f_{S}(\mu)$, so

$$
f_{S}(\mu)=\left\{\mu^{\prime} \in \mathcal{M} \mid \mu^{\prime}(S)=S \text { and } \mu^{\prime} \succ_{S} \mu\right\}
$$

We say that the matching $\mu^{\prime}$ dominates the matching $\mu$ if there is a coalition $S \in \mathcal{N}$ such that $\mu^{\prime}$ dominates the matching $\mu$ via $S$ and we denote the set of all such matchings by $f(\mu)$, so

$$
f(\mu)=\cup_{S \in \mathcal{N}} f_{S}(\mu) .
$$

The core $C$ of the matching problem $(M, W, \succ)$ consists of all matchings that are not blocked by any coalition, so

$$
C=\{\mu \in \mathcal{M} \mid f(\mu)=\emptyset\}
$$

It has been shown by Gale and Shapley (1962) that the core of a matching problem is non-empty. Also, a matching is stable if and only if it is not blocked by a coalition of size one or two and if and only if it belongs to the core, see Theorem 3.3 in Roth and Sotomayor (1990).

We next present the standard definition of a von Neumann Morgenstern (vNM) stable set as introduced in von Neumann and Morgenstern (1944) when applied to matching problems.

Definition 1. A set of matchings $V \subset \mathcal{M}$ is a vNM stable set if it satisfies:

(i) Internal stability: For every $\mu, \mu^{\prime} \in V$, it holds that $\mu^{\prime} \notin f(\mu)$.

(ii) External stability: For every $\mu \in \mathcal{M} \backslash V$, it holds that $f(\mu) \cap V \neq \emptyset$. 
A set of matchings is a vNM stable set if it satisfies internal and external stability. Internal stability requires that no matching inside the vNM stable set is dominated by a matching belonging to the vNM stable set. External stability states that any matching outside the vNM stable set is dominated by some matching that belongs to the vNM stable set.

As for the definition of the core, there is no loss of generality to restrict attention to coalitions of size one and two when formulating internal and external stability or to restrict attention to pairwise deviations. It is immediate that the core is contained in any vNM stable set. However, since the core may not satisfy external stability, it may not be a vNM stable set itself.

Given two matchings $\mu, \mu^{\prime} \in \mathcal{M}$, let $\mu \vee \mu^{\prime}: N \rightarrow N$ be the function defined by

$$
\begin{array}{rll}
\left(\mu \vee \mu^{\prime}\right)(m) & =\max _{\succ_{m}}\left\{\mu(m), \mu^{\prime}(m)\right\}, & m \in M, \\
\left(\mu \vee \mu^{\prime}\right)(w) & =\min _{\succ_{w}}\left\{\mu(w), \mu^{\prime}(w)\right\}, & w \in W .
\end{array}
$$

Similarly, we define $\mu \wedge \mu^{\prime}: N \rightarrow N$ as

$$
\begin{aligned}
\left(\mu \wedge \mu^{\prime}\right)(m) & =\min _{\succ_{m}}\left\{\mu(m), \mu^{\prime}(m)\right\}, \quad m \in M \\
\left(\mu \wedge \mu^{\prime}\right)(w) & =\max _{\succ_{w}}\left\{\mu(w), \mu^{\prime}(w)\right\}, \quad w \in W .
\end{aligned}
$$

In general, the functions $\mu \vee \mu^{\prime}$ and $\mu \wedge \mu^{\prime}$ need not be matchings.

A set $X \subset \mathcal{M}$ of matchings is a lattice if for every $\mu, \mu^{\prime} \in X$ it holds that $\mu \vee \mu^{\prime} \in X$ and $\mu \wedge \mu^{\prime} \in X$. The set $X$ is a distributive lattice if $X$ is a lattice and for every $\mu, \mu^{\prime}, \mu^{\prime \prime} \in X$ it holds that

$$
\begin{aligned}
& \mu \vee\left(\mu^{\prime} \wedge \mu^{\prime \prime}\right)=\left(\mu \vee \mu^{\prime}\right) \wedge\left(\mu \vee \mu^{\prime \prime}\right) \\
& \mu \wedge\left(\mu^{\prime} \vee \mu^{\prime \prime}\right)=\left(\mu \wedge \mu^{\prime}\right) \vee\left(\mu \wedge \mu^{\prime \prime}\right)
\end{aligned}
$$

Knuth (1976) has shown that the core $C$ of a matching problem is a distributive lattice. Ehlers (2007) has shown that $V$ is a vNM stable set only if $V$ is a maximal set satisfying $C \subset V, V$ is a distributive lattice, and the set of single agents is the same for every element of $V$. Moreover, $V$ is a vNM stable set if $V$ is the unique maximal set satisfying $C \subset V$, $V$ is a distributive lattice, and the set of single agents is the same for every element of $V$. Thus, a vNM stable set $V$ contains the core but can also contain matchings outside the core.

\section{Myopic vNM Sets}

Ray and Vohra (2015) express the following criticism towards the vNM stable set for nontransferable utility games: the enforceability condition embedded in the definition of the dominance relation of vNM stable sets denies the coalitional sovereignty of players outside 
the objecting coalition. When adapted to matchings, their objection is as follows. Since in the formulation of the vNM stable set using the dominance relation $f$, a coalition $S \in \mathcal{N}$ can impose any matching as long as its restriction to $S$ is feasible for $S$, the objecting coalition is given implicitly the power to arrange the matchings of players outside the coalition, which is clearly undesirable.

In the following, we adapt the dominance relation in such a way that coalitional sovereignty of players outside the objecting coalition is respected. Contrary to the dominance relation $f$, it may now matter whether the size of the objecting coalition is unrestricted or takes place by individuals and blocking pairs. We therefore formulate two dominance relations.

Assume first that we do not restrict the size of the objecting coalition. In order to respect the coalitional sovereignty property, the blocking of a matching $\mu \in \mathcal{M}$ by a coalition $S \in \mathcal{N}$ should result in a matching $\mu^{\prime}$ such that the players $i \in \mu(S) \backslash S$ become single, whereas the players in $N \backslash(S \cup \mu(S))$ are not affected by this blocking. ${ }^{2}$ We therefore restrict attention to matchings $\mu^{\prime}$ with this feature and define the set of matchings that dominate $\mu$ via $S$ as follows:

$$
\begin{aligned}
g_{S}(\mu)=\left\{\mu^{\prime} \in \mathcal{M} \mid\right. & \mu^{\prime}(S)=S, \\
& \text { for every } i \in \mu(S) \backslash S, \mu^{\prime}(i)=i, \\
& \text { for every } i \in N \backslash(S \cup \mu(S)), \mu^{\prime}(i)=\mu(i), \\
& \left.\mu^{\prime} \succ_{S} \mu\right\} .
\end{aligned}
$$

It clearly holds that $g_{S}(\mu) \subset f_{S}(\mu)$. The set of matchings that dominate $\mu$ is now defined as follows:

$$
g(\mu)=\cup_{S \in \mathcal{N}} g_{S}(\mu)
$$

We refer to an element of $g(\mu)$ as a myopic improvement.

In matching theory it is typical to restrict blocking to individuals and blocking pairs. Let some matching $\mu \in \mathcal{M}$ be given. An individual $i \in N$ can destroy his match with player $\mu(i)$ whenever $\mu(i) \neq i$. The players in $N \backslash\{i, \mu(i)\}$ are not affected by the destruction of this match, so remain matched to the same partner. The resulting matching is denoted by $\mu^{\prime}=\mu-(i, \mu(i))$.

A blocking pair $(m, w)$ can destroy their existing matches, if any, and form a match between them. The players in $N \backslash\{m, w, \mu(m), \mu(w)\}$ are not affected by the formation of such a match, so keep their existing partners. The resulting matching is denoted by $\mu^{\prime}=\mu+(m, w)$.

\footnotetext{
${ }^{2}$ Notice that this enforceability condition is similar to the enforceability condition defined in Roth and Sotomayor (1990).
} 
Let some matching $\mu \in \mathcal{M}$ and some coalition $S \in \mathcal{N}$ with $S=\{i\}$ for some $i \in N$ or $S=\{m, w\}$ with $m \in M$ and $w \in W$ be given. We define the set of matchings that dominate $\mu$ via $S$ as follows:

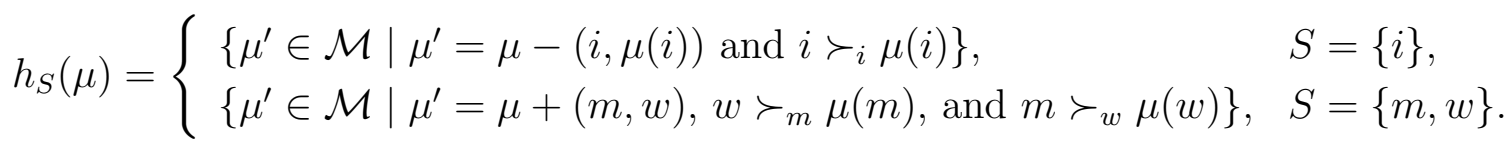

Observe that for singleton coalitions $S$ it holds that $h_{S}(\mu)=g_{S}(\mu)$ and for coalitions $S$ consisting of a man and a woman we have $h_{S}(\mu) \subset g_{S}(\mu)$. In the latter case, inequality may result because under $h_{S}$ it is not allowed that $m$ and $w$ destroy their current match without forming a new one together. Notice that in all cases, $h_{S}(\mu)$ is either the empty set or a singleton. We define the set of matchings that dominate $\mu$ as follows:

$$
h(\mu)=\left(\cup_{i \in N} h_{\{i\}}(\mu)\right) \cup\left(\cup_{(m, w) \in M \times W} h_{\{m, w\}}(\mu)\right) .
$$

We refer to an element of $h(\mu)$ as a pairwise myopic improvement.

Although it might be tempting to define the vNM stable set by replacing $f(\mu)$ by either $g(\mu)$ or $h(\mu)$ in Definition 1, the following example shows this to be problematic.

Example 1. Consider a matching problem $(M, W, \succ)$, where $M=\left\{m_{1}, m_{2}\right\}, W=$ $\left\{w_{1}, w_{2}\right\}$, and the preferences of the individuals are as follows.

$\begin{array}{ccccc}\frac{m_{1}}{w_{1}} & \frac{m_{2}}{w_{1}} & & \frac{w_{1}}{m_{1}} & \frac{w_{2}}{m_{2}} \\ w_{2} & w_{2} & & m_{2} & m_{1} \\ m_{1} & m_{2} & & w_{1} & w_{2}\end{array}$

We represent a matching $\mu$ by the set of $(m, w)$ pairs that are matched in $\mu$. There is a single stable matching $\mu^{*}=\left\{\left(m_{1}, w_{1}\right),\left(m_{2}, w_{2}\right)\right\}$. The core has $\mu^{*}$ as its unique element. For every $\mu \in \mathcal{M}$ such that $\mu \neq \mu^{*}$ we have that $\mu^{*} \in f(\mu)$. It now follows easily that there is a unique vNM stable set $V_{f}$ and that this set is equal to the core, $V_{f}=C=\left\{\mu^{*}\right\}$.

However, according to the dominance relation $g$, the matching $\mu^{*}$ does not dominate the matching $\mu^{\prime}=\left\{\left(m_{1}, w_{2}\right),\left(m_{2}, w_{1}\right)\right\}$. Indeed, only the grand coalition $N$ can enforce the matching $\mu^{*}$ from $\mu^{\prime}$ under $g$. Since $\mu^{\prime} \succ_{m_{2}} \mu^{*}, m_{2}$ would block the deviation by the grand coalition from $\mu^{\prime}$ to $\mu^{*}$.

Let $V_{g}$ be a vNM stable set based on the dominance relation $g$. Since $g\left(\mu^{*}\right)=\emptyset$, external stability requires $\mu^{*} \in V_{g}$. For every matching $\mu \in \mathcal{M} \backslash\left\{\mu^{*}, \mu^{\prime},\left\{\left(m_{2}, w_{1}\right)\right\}\right\}$, it holds that $\mu^{*} \in g(\mu)$, so in order not to violate internal stability, $\mu \notin V_{g}$. Since $g\left(\mu^{\prime}\right)=\left\{\left\{\left(m_{1}, w_{1}\right)\right\}\right\}$ and $\left\{\left(m_{1}, w_{1}\right)\right\} \notin V_{g}$, it holds that $\mu^{\prime} \in V_{g}$ by external stability. Since $\mu^{\prime} \in g\left(\left\{\left(m_{2}, w_{1}\right)\right\}\right)$, it follows that $V_{g}=\left\{\mu^{*}, \mu^{\prime}\right\}$. 
The fact that $\mu^{\prime}$ belongs to $V_{g}$ is problematic. The only myopic improvement from $\mu^{\prime}$ is given by the matching $\left\{\left(m_{1}, w_{1}\right)\right\}$. Since $\left\{\left(m_{1}, w_{1}\right)\right\}$ does not belong to $V_{g}$, one needs $\mu^{\prime}$ to be part of $V_{g}$ in order to satisfy external stability. However, starting from the matching $\left\{\left(m_{1}, w_{1}\right)\right\}$, there is only one further myopic improvement, i.e. to $\mu^{*}$, and from $\mu^{*}$ there are no further myopic improvements. Given a matching $\mu^{\prime}$, one would therefore expect to end up in $\mu^{*}$, which is part of $V_{g}$, and $\mu^{\prime}$ should not be regarded as stable.

When using the dominance relation $h$ in Definition 1, the sets $\left\{\mu^{*}\right\}$ and $\left\{\mu^{*}, \mu^{\prime}\right\}$ would not qualify as stable sets. Denoting the matching where every individual is unmatched by $\mu^{\emptyset}$, we have that $\mu^{*} \notin h\left(\mu^{\emptyset}\right)$ and $\mu^{\prime} \notin h\left(\mu^{\emptyset}\right)$, so external stability would be violated.

Let $V_{h}$ be a vNM stable set based on the dominance relation $h$. Since $h\left(\mu^{*}\right)=\emptyset$, external stability requires $\mu^{*} \in V_{h}$. Since $\mu^{*} \in h\left(\left\{\left(m_{1}, w_{1}\right)\right\}\right)$ and $\mu^{*} \in h\left(\left\{\left(m_{2}, w_{2}\right)\right\}\right)$, it follows from internal stability that $\left\{\left(m_{1}, w_{1}\right)\right\} \notin V_{h}$ and $\left\{\left(m_{2}, w_{2}\right)\right\} \notin V_{h}$.

Since $h\left(\mu^{\prime}\right)=\left\{\left\{\left(m_{1}, w_{1}\right)\right\}\right\}$ and $\left\{\left(m_{1}, w_{1}\right)\right\} \notin V_{h}$, it follows from external stability that $\mu^{\prime} \in V_{h}$.

Since $h\left(\mu^{\emptyset}\right)=\left\{\left\{\left(m_{1}, w_{1}\right)\right\},\left\{\left(m_{2}, w_{2}\right)\right\}\right\}$ and $\left\{\left(m_{1}, w_{1}\right)\right\},\left\{\left(m_{2}, w_{2}\right)\right\} \notin V_{h}$, external stability requires that $\mu^{\emptyset} \in V_{h}$.

Finally, since $\mu^{\prime} \in h\left(\left\{\left(m_{1}, w_{2}\right)\right\}\right)$ and $\mu^{\prime} \in h\left(\left\{\left(m_{2}, w_{1}\right)\right\}\right)$, it follows from internal stability that $\left\{\left(m_{1}, w_{2}\right)\right\} \notin V_{h}$ and $\left\{\left(m_{2}, w_{1}\right)\right\} \notin V_{h}$.

We reach the conclusion that $V_{h}=\left\{\mu^{*}, \mu^{\prime}, \mu^{\emptyset}\right\}$ is the unique vNM stable set based on the dominance relation $h$. Since $\mu^{\emptyset}$ is the worst matching for all individuals, this is a highly undesirable conclusion.

Example 1 shows that the assumption of coalitional sovereignty affects the vNM stable set. Also, under coalitional sovereignty, it matters whether blocking coalitions can be of any size or is blocking is restricted to pairwise deviations. The reason for this difference is that external stability requires each matching not in the stable set to be directly dominated by an element in the stable set. If a matching $\mu$ is dominated by another matching $\mu^{\prime}$ via a coalition $S$ of cardinality greater than two, then it may not be the case that $\mu^{\prime}$ can be enforced from $\mu$ by an individual or a pair.

Rather than requiring direct domination, it should be sufficient for external stability that there is a sequence of matchings, each matching in the sequence dominating the previous one, that ultimately reaches a matching in the stable set. Indeed, if a matching that is not in the stable set is blocked by some coalition and the resulting matching is not stable itself, then further improvements will take place. We make a similar requirement for testing internal stability. That is, if a matching belongs to the stable set, then there should be no sequence of matchings, each matching in the sequence being dominated by the next one that ultimately reaches another matching in the stable set. 
Let some $\mu \in \mathcal{M}$ be given. The set

$$
h^{2}(\mu)=h(h(\mu))=\left\{\mu^{\prime \prime} \in \mathcal{M} \mid \exists \mu^{\prime} \in h(\mu) \text { such that } \mu^{\prime \prime} \in h\left(\mu^{\prime}\right)\right\}
$$

consists of those matchings that can be reached by a composition of two pairwise myopic improvements starting at $\mu$. We extend this definition and, for $k \in \mathbb{N}$, we define $h^{k}(\mu)$ as those matchings that can be reached from $\mu$ by a composition of $k$ pairwise myopic improvements. Since the set $\mathcal{M}$ is finite, it follows that for some $k^{\prime} \in \mathbb{N}$, for every $k \geq k^{\prime}$, for every $\mu \in \mathcal{M}, h^{k}(\mu)=h^{k^{\prime}}(\mu)$. The resulting relation is called the transitive closure of $h$ and is denoted by $h^{\infty}$.

Definition 2. A set of matchings $V \subset \mathcal{M}$ is a pairwise myopic vNM set if it satisfies:

(i) Pairwise myopic internal stability: For every $\mu, \mu^{\prime} \in V$, it holds that $\mu^{\prime} \notin h^{\infty}(\mu)$.

(ii) Pairwise myopic external stability: For every $\mu \in \mathcal{M} \backslash V$, it holds that $h^{\infty}(\mu) \cap V \neq \emptyset$.

Condition (i) of Definition 2 corresponds to pairwise myopic internal stability. For any two matchings $\mu$ and $\mu^{\prime}$ in the pairwise myopic vNM set $V$ it does not hold that there is a sequence of myopic improvements from one to the other. Condition (ii) of Definition 2 expresses pairwise myopic external stability. For every matching $\mu$ outside the pairwise myopic vNM set $V$ it holds that there is $\mu^{\prime} \in V$ and a sequence of matchings $\mu_{0}, \ldots, \mu_{k^{\prime}}$ with $\mu_{0}=\mu$ and $\mu_{k^{\prime}}=\mu^{\prime}$ such that, for every $k \in\left\{0, \ldots, k^{\prime}-1\right\}, \mu_{k+1} \in h\left(\mu_{k}\right)$.

The definition of a pairwise myopic vNM set is equivalent to the definition of a generalized stable set for abstract systems as introduced in van Deemen (1991), when we consider the abstract system $(\mathcal{M}, h)$. It corresponds to the stable set with respect to path dominance in Page and Wooders (2009) when $h$ is taken to be the direct dominance relation.

Theorem 1. A set of matchings is a pairwise myopic vNM set if and only if it is equal to the core.

Proof. We start with the "if" part of the proof and show that the core $C$ is a pairwise myopic vNM set.

Let some $\mu \in C$ be given. It holds by definition of the core that $f(\mu)=\emptyset$. Since $h(\mu) \subset f(\mu)$, it follows that $h(\mu)=\emptyset$. Pairwise myopic internal stability follows.

Let some $\mu \in \mathcal{M} \backslash C$ be given. We have to show that $h^{\infty}(\mu) \cap C \neq \emptyset$. It follows from Theorem in Roth and Vande Vate $(1990)$ that $h^{\infty}(\mu)$ contains a stable matching. Since a stable matching belongs to $C$ by Theorem 3.3 in Roth and Sotomayor (1990), pairwise myopic external stability follows.

We continue with the "only if" part of the proof and show that $C$ is the only pairwise myopic vNM set. Let $V$ be a pairwise myopic vNM set. For every $\mu \in C$ it holds that 
$h(\mu) \subset f(\mu)=\emptyset$. Since $V$ satisfies pairwise myopic external stability, it follows that $C \subset V$.

Suppose $V \backslash C \neq \emptyset$. Let some $\mu^{\prime} \in V \backslash C$ be given. Combining again Theorem in Roth and Vande Vate (1990) and Theorem 3.3 in Roth and Sotomayor (1990), it follows that $h^{\infty}\left(\mu^{\prime}\right) \cap C \neq \emptyset$, so therefore $h^{\infty}\left(\mu^{\prime}\right) \cap V \neq \emptyset$, thereby violating pairwise myopic internal stability. Consequently, it holds that $V \backslash C=\emptyset$.

Knuth (1976) observes that the process of allowing blocking pairs to form may lead to cycles of blocking pairs and then may not lead to a stable matching. By means of an example, Knuth (1976) raises the question of whether, for any preferences of the agents, there exists at least one path from any matching to a stable matching. Theorem of Roth and Vande Vate (1990) resolves this question and proposes a family of random processes, beginning from an arbitrary matching and selecting a blocking pair at random to create a new matching, that will reach a stable matching with probability one. In the proof of Theorem 1, we use the result by Roth and Vande Vate (1990) to prove that pairwise myopic external stability follows and that the core is the unique pairwise myopic vNM set.

An alternative to the pairwise myopic vNM set is to allow a coalition of any size to form and block, while respecting coalitional sovereignty. We denote the transitive closure of $g$ by $g^{\infty}$.

Definition 3. A set of matchings $V \subset \mathcal{M}$ is a myopic vNM set if it satisfies:

(i) Myopic internal stability: For every $\mu, \mu^{\prime} \in V$, it holds that $\mu^{\prime} \notin g^{\infty}(\mu)$.

(ii) Myopic external stability: For every $\mu \in \mathcal{M} \backslash V$, it holds that $g^{\infty}(\mu) \cap V \neq \emptyset$.

The definition of a myopic vNM set is equivalent to the definition of a generalized stable set for abstract systems as introduced in van Deemen (1991), when we consider the abstract system $(\mathcal{M}, g)$ and it corresponds to the stable set with respect to path dominance in Page and Wooders (2009) when $g$ is taken to be the direct dominance relation. The next result demonstrates that allowing larger coalitions to form and block does not change our previous result: the unique myopic vNM stable set in a one-to-one matching market coincides with the core.

Theorem 2. For every $\mu \in \mathcal{M}$ it holds that $g^{\infty}(\mu)=h^{\infty}(\mu)$.

Proof. Let some $\mu \in \mathcal{M}$ be given. Obviously, it holds that $h^{\infty}(\mu) \subset g^{\infty}(\mu)$.

To show the converse, it is sufficient to show that for every $\mu \in \mathcal{M}$, for every $\mu^{\prime} \in g(\mu)$, there is a sequence $\mu_{0}, \ldots, \mu_{k^{\prime}}$ such that $\mu=\mu_{0}, \mu^{\prime}=\mu_{k^{\prime}}$ and, for every $k=0, \ldots, k^{\prime}-1$, $\mu_{k+1} \in h\left(\mu_{k}\right)$. 
Let $\mu \in \mathcal{M}, \mu^{\prime} \in g(\mu)$, and $S \in \mathcal{N}$ be given such that $\mu^{\prime}(S)=S$, for every $i \in \mu(S) \backslash S$, $\mu^{\prime}(i)=i$, for every $i \in N \backslash(S \cup \mu(S)), \mu^{\prime}(i)=\mu(i)$, and $\mu^{\prime} \succ_{S} \mu$. The set $S$ can be partitioned into sets $S_{1}=\left\{i_{1}, \ldots, i_{j^{\prime}}\right\}$ and $S_{2}=\left\{i_{j^{\prime}+1}, \ldots, i_{2 k^{\prime}-j^{\prime}}\right\}$ such that for every $i \in S_{1}, \mu^{\prime}(i)=i$ and, for every $i_{k} \in S_{2}$ with $k \leq k^{\prime}, \mu^{\prime}\left(i_{k}\right)=i_{k+k^{\prime}-j^{\prime}}$. We form the sequence $\mu_{0}, \ldots, \mu_{k^{\prime}}$ by defining $\mu_{k+1}=\mu_{k}-\left(i_{k+1}, \mu\left(i_{k+1}\right)\right)$ for every $k=0, \ldots, j^{\prime}-1$, and $\mu_{k+1}=\mu_{k}+\left(i_{k+1}, \mu^{\prime}\left(i_{k+1}\right)\right)$ for every $k=j^{\prime}, \ldots, k^{\prime}-1$. It is easily verified that, for every $k=0, \ldots, k^{\prime}-1$, it holds that $\mu_{k+1} \in h\left(\mu_{k}\right)$.

The following result now follows as an easy corollary.

Corollary 1. A set of matchings is a myopic vNM set if and only if it is equal to the core.

In general, the vNM stable set with $g$ as the dominance relation, $V_{g}$, is substantially different from the vNM stable set with $h$ as the dominance relation, $V_{h}$, as shown in Example 1. However, these differences disappear whenever the external stability as based on direct dominance is replaced by a condition that allows for a sequence of matchings, each matching in the sequence being dominated by the next one that ultimately reaches a matching in the set.

\section{Level-1 Farsighted Sets}

An alternative to the pairwise myopic vNM set and the myopic vNM set is the pairwise myopically stable set as defined in Herings, Mauleon, and Vannetelbosch (2009) for network formation problems. This concept is equivalent to the level-1 farsighted set of Herings, Mauleon, and Vannetelbosch (2014), who also define this notion for higher levels of farsightedness. Their definitions for network formation problems can be adapted in a straightforward way to one-to-one matching problems.

Definition 4. A set of matchings $U \subset \mathcal{M}$ is a level-1 farsighted set if it satisfies:

(i) Level-1 deterrence of external deviations: For every $\mu \in U, h(\mu) \cap(\mathcal{M} \backslash U)=\emptyset$.

(ii) Level-1 external stability: For every $\mu \in \mathcal{M} \backslash U$, it holds that $h^{\infty}(\mu) \cap U \neq \emptyset$.

(iii) Minimality: There is no proper subset of $U$ satisfying Conditions (i) and (ii).

Condition (i) in Definition 4 captures level-1 deterrence of external deviations and requires that there is no pairwise myopic improvement from a matching $\mu \in U$ to a matching outside $U$. Condition (ii) requires level-1 external stability and coincides with pairwise myopic external stability in Definition 2. It requires that, for every matching $\mu \in \mathcal{M} \backslash U$ outside the level-1 farsighted set, there exists a sequence of matchings, each matching in 
the sequence being a pairwise myopic improvement of the previous one, that ultimately reaches a matching in the set. Notice that the set of all matchings $\mathcal{M}$ trivially satisfies Conditions (i) and (ii) in Definition 4. This motivates Condition (iii), the minimality condition. That is, there is no proper subset of $U$ satisfying the first two conditions. As has also been argued in Myerson and Weibull (2015), minimality can also be viewed as a requirement of internal stability.

For network formation problems, Herings, Mauleon, and Vannetelbosch (2014) show that there always exists a unique level-1 farsighted set. It is given by the set consisting of all networks that belong to closed cycles, so it includes all pairwise stable networks. In the following, we characterize the level-1 farsighted sets for one-to-one matching problems. Notice that both the level-1 farsighted set and the pairwise myopic vNM set require level-1 external stability, also called pairwise myopic external stability. On top of this, the level-1 farsighted set requires level-1 deterrence of external deviations and minimality, and the pairwise myopic vNM set requires pairwise myopic internal stability. We show now that, for one-to-one matching problems, both solutions concepts coincide with the core.

Theorem 3. A set of matchings is a level-1 farsighted set if and only if it is equal to the core.

Proof. We show first that the core $C$ is a level-1 farsighted set.

Let some $\mu \in C$ be given. It holds by definition of the core that $f(\mu)=\emptyset$. Since $h(\mu) \subset f(\mu)$, it follows that $h(\mu)=\emptyset$. Level-1 deterrence of external deviations follows.

Let some $\mu \in \mathcal{M} \backslash C$ be given. Since level-1 external stability is equivalent to pairwise myopic external stability, Condition (ii) of Definition 4 follows from Theorem 1.

For every $\mu \in C$ it holds that $h(\mu)=\emptyset$, so $h^{\infty}(\mu)=\emptyset$. Level-1 external stability therefore requires $C$ to be a subset of every level- 1 farsighted set. It follows that $C$ satisfies Condition (iii) of Definition 4.

We have shown $C$ to be a level-1 farsighted set. Since we also argued $C$ to be contained in every level-1 farsighted set in order to satisfy level-1 external stability, the minimality condition implies it to be the unique level-1 farsighted set.

The notion of a level-1 farsighted set can also be defined when arbitrary coalitional deviations are allowed for, so by replacing the dominance relation $h$ by the dominance relation $g$ in Definition 4 . Let $U \subset \mathcal{M}$ be any set of matchings satisfying the alternative of Definition 4 based on the dominance relation $g$. Since $g(\mu)=\emptyset$ for every $\mu \in C$, level-1 external stability requires the core $C$ to be contained in $U$. Since $g(\mu)=\emptyset, C$ satisfies the version of Condition (i) of Definition 4 as based on $g$. Since $h^{\infty}(\mu) \subset g^{\infty}(\mu)$ for every $\mu \in \mathcal{M}$, Theorem 3 implies that $C$ satisfies Condition (ii) of Definition 4 as based on $g$. From minimality, we conclude that $U=C$. 


\section{Conclusion}

We propose different notions of stable sets for one-to-one matching problems that take the requirement of coalitional sovereignty into account. Coalitional sovereignty means that the objecting coalition does not have the power to arrange the matchings of agents outside the coalition. In these new notions of stable sets, the internal and external stability conditions are formulated using path dominance, following van Deemen (1991) and Page and Wooders (2009), and motivated by Example 1. If only pairwise deviations are allowed, then path dominance corresponds to the myopic blocking dynamics introduced by Roth and Vande Vate (1990) for marriage markets.

We consider both general coalitional deviations as well as pairwise deviations, resulting in the concepts of myopic vNM set and pairwise myopic vNM set. We obtain a unique prediction: the matchings that belong to the core. Thus, while Ehlers (2007) has shown that the set of matchings in the core is a subset of any vNM stable set and may contain matchings outside the core when the enforceability condition does not respect coalitional sovereignty, we obtain the same predictions as Roth and Vande Vate (1990) and Jackson and Watts (2002): both the pairwise myopic vNM set and the myopic vNM set concide with the core.

We also adapt the level-1 farsighted set of Herings, Mauleon, and Vannetelbosch (2014), defined for general network formation problems, to one-to-one matching problems. We again obtain the core as the unique prediction, both when only pairwise deviations are allowed as well as under general coalitional deviations. Mauleon, Vannetelbosch, and Vergote (2011) have found the singleton core elements as the stable set prediction under farsighted behavior of the agents. Here we show that the same conclusion follows if agents are myopic.

\section{Acknowledgments}

Vincent Vannetelbosch and Ana Mauleon are Senior Research Associates of the National Fund for Scientific Research (FNRS). Financial support from the Spanish Ministry of Economy and Competition under the project ECO2015-64467-R, from the Fonds de la Recherche Scientifique - FNRS under the grant J.0073.15 and from the Belgian French speaking community ARC project $n^{\circ} 15 / 20-072$ of Saint-Louis University - Brussels is gratefully acknowledged.

\section{References}

Chwe, M. S.-Y. (1994), "Farsighted Coalitional Stability," Journal of Economic Theory, 63, 299-325. 
Deemen, A.M.A. van (1991), "A Note on Generalized Stable Sets," Social Choice and Welfare, 8, 255-260.

Ehlers, L. (2007), "Von Neumann-Morgenstern Stable Sets in Matching Problems," Journal of Economic Theory, 134, 537-547.

Gale, D., and L.S. Shapley (1962), "College Admissions and the Stability of Marriage," American Mathematical Monthly, 69, 9-15.

Harsanyi, J.C. (1974), "An Equilibrium-point Interpretation of Stable Sets and a Proposed Alternative Definition," Management Science, 20, 1472-1495.

Herings, P.J.J., A. Mauleon, and V. Vannetelbosch (2009), "Farsightedly Stable Networks," Games and Economic Behavior, 67, 526-541.

Herings, P.J.J., A. Mauleon, and V. Vannetelbosch (2014), "Stability of Networks under Level-K Farsightedness," METEOR Research Memorandum 14/30, Maastricht University, Maastricht, pp. 1-39.

Jackson, M.O., and A. Watts (2002), "The Evolution of Social and Economic Networks," Journal of Economic Theory, 106, 265-295.

Knuth, D.E. (1976), Marriages Stables, Les Presses de l'Université de Montreal, Montreal.

Mauleon, A., V. Vannetelbosch, and W. Vergote (2011), "Von Neumann-Morgenstern Farsightedly Stable Sets in Two-sided Matching," Theoretical Economics, 6, 499-521.

Myerson, R.B., and J. Weibull (2015), "Tenable Strategy Blocks and Settled Equilibria," Econometrica, 83, 943-976.

Neumann, J. von, and O. Morgenstern (1944), Theory of Games and Economic Behavior, Princeton University Press, Princeton, New Jersey.

Page, F.H., Jr., and M. Wooders (2009), "Strategic Basins of Attraction, the Path Dominance Core, and Network Formation Games," Games and Economic Behavior, $66,462-487$.

Ray, D., and R. Vohra (2015), "The Farsighted Stable Set," Econometrica, 83, 9771011.

Roth, A.E., and M.A.O. Sotomayor (1990), Two-sided Matching, A Study in Gametheoretic Modeling and Analysis, Econometric Society Monographs No. 18, Cambridge University Press, Cambridge. 
Roth, A.E., and J.H. Vande Vate (1990), "Random Paths to Stability in Two-sided Matching," Econometrica, 58, 1475-1480.

Shapley, L.S. (1971), "Cores of Convex Games," International Journal of Game Theory, $1,11-26$. 